\title{
Design of a 5-dof Haptic Simulator for Urological Operations
}

\author{
Evangelos Papadopoulos ${ }^{1}$, Kostas Vlachos ${ }^{1}$ and Dionyssios Mitropoulos ${ }^{2}$ \\ ${ }^{1}$ Department of Mechanical Engineering, National Technical University of Athens, 15780 Athens, Greece \\ egpapado@central.ntua.gr, kostaswl@central.ntua.gr \\ ${ }^{2}$ School of Medicine, National Capodistrian University of Athens, Athens, Greece
}

\begin{abstract}
A new haptic feedback mechanism with five active degrees of freedom (dof), part of a training simulator for urological operations, is presented. The mechanism consists of a 2-dof 5-bar linkage, and a 3dof spherical joint. To reproduce very small forces and moments, the mechanism has low friction, inertia and mass, is statically balanced, and has a simple mass matrix. Roll-pitch-yaw motions of the tool result in motions of the corresponding actuator. Force feedback transmission is achieved via capstan drives and idler pulleys. The computation of the currents and the structure of the control loop are described.
\end{abstract}

\section{INTRODUCTION}

Research on the use of virtual reality in medicine has rapidly increased due to developments in software and hardware, [1]. Training in a simulated environment has significant advantages against traditional training. It is a less expensive, it reduces animals use which has become undesirable for ethical and economical reasons, and results in faster training in complicated procedures, [2].

Development of a useful and realistic surgical simulator requires the use of a virtual reality graphical environment and of haptic technologies including force feedback. While realistic visual representation of human anatomy and tissue deformation is very important, the ability to interact with the simulated tissues through the feel of forces and torques is also of paramount importance. In order to implement such a feel, haptic devices are used. Although early haptic devices were of the master - slave type, [3], today, the slave system has been replaced by a computational model.

Two approaches exist in the development of medical simulators. The first uses general purpose haptic devices, like the Phantom or the Freedom7, [4, 5, 6]. The Phantom is a five bar parallel mechanism with three or six degrees-of-freedom (dof) which is designed as a general-use haptic interface, [7]. The Phantom 1.5/6DOF and the Phantom Premium 6DOF Prototype have six active dof, and are designed mainly for virtual prototyping, virtual assembly, etc. The actuators are mounted on moving parts of the mechanism, while its inertia and force range are not suitable for urological operation simulators, [8]. A training simulator for the diagnosis of prostate cancer using the Phantom haptic interface is described in [9]. Freedom 7 is a haptic device with six dof for translation and rotation, plus a seventh for the movement of tools like scissors and forceps. It has six active dofs but it is also designed for general haptic applications, [10].

The second approach uses haptic devices designed for a specific operation. The PantoScope, a four dof mechanism for simulating a laparoscopic operation, belongs to this category, [11]. The Laparoscopic Impulse Engine is a five dof, three active dof for laparoscopic and endoscopic procedures. VIRGY is an endoscopic surgery simulator, which uses the PantoScope haptic device, [12]. The Karlsruhe Endoscopic Surgery Trainer and the VEST are training simulators specialized in laparoscopic operations using the KISMET software, [13].

This paper presents a new five dof haptic device for male urological operations, of which all five dof are active. The mechanism allows three dof tool orientation motions in large ranges and two dof translation motions. Roll-pitch-yaw motions of the tool result in motions of the corresponding actuator. Unlike other haptic devices in which large forces or torques are of prime importance, this device is designed to reproduce faithfully very small forces and torques, like those that appear in urological operations. A major effort was placed in designing the mechanism with low friction and inertia. To this end, motors are fixed in the base and capstan drives are employed. The paper analyzes the kinematics and the dynamics of the mechanism, and describes its mechanical design. It proposes a control law and discusses implementation solutions.

\section{MECHANICAL REQUiREMENTS \& SyNTHESIS}

In urological operations, a long cylindrical endoscope, see Fig. 1, is first inserted in a patient till the endpoint reaches the bladder. A tiny camera provides a view of the tissues through a monitor. The surgeon, using a scissorlike mechanism, moves the tip of the end-point to cut tissues at various locations and provides electric current activated by a foot pedal.

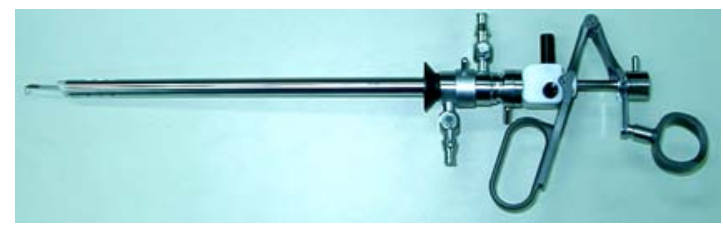

Fig. 1. Endoscope for urological operations. 
The forces and torques experienced by the surgeon are very small. However, these provide very important clues about the progress of the operation.

Currently, training for such operations is done mostly in vivo animals, such as pigs. A simulator can provide the necessary training, reduce the need for animals, and enhance training capabilities by measuring trainee performance and by providing customized human anatomies or maladies. Since in urological operations visual feedback is through a monitor while haptic feedback is provided by the endoscope, these operations are well-suited for the development of a training simulator. Such a system must consist of a haptic device to represent the endoscope and its interactions, and a graphical simulator to depict synthetic images that would be provided by the real endoscope camera.

To design the haptic interface, a series of observations and experiments were conducted. Due to the difficulty to transmit surgeon experience to an engineering team, operations on humans were observed to acquire a sense of the required workspace. Also, the engineering team participated in an in-vivo animal operation and experienced forces and torques fed back by the endoscope.

Observations showed that from the motion point of view, a urological operation can be divided in two phases, the insertion and the main operation. During insertion, the surgeon moves the tip of the endoscope along a path from the insertion point $\mathrm{A}$ to the final point $\mathrm{C}$, via an intermediate point $\mathrm{B}$ as shown in Fig. 2. At point $\mathrm{B}$, the endoscope orientation changes without translation, so as to align the entire urethra and continue the insertion without traumas. The corresponding endoscope poses are labeled by a, b, c, d, and are shown in Fig. 2. During the main operation, the endoscope rotates in all directions but its tip translates minimally. These observations showed that although the endoscope can have any orientation in a cone, its tip translations occur on a plane of symmetry of the patient. Therefore, a five-dof haptic device is needed, with two translational and three rotational dof.

The actual kinematic requirements that define the workspace of the haptic interface are shown in Table 1. The workspace is defined with respect to a reference coordinate frame $(\mathrm{O}, \mathrm{X}, \mathrm{Y}, \mathrm{Z})$, where $\mathrm{O}$ is the base point of the mechanism and $\mathrm{X}-\mathrm{Y}$ the plane of symmetry of the patient. The rotations are defined with respect to an endoscope body-fixed coordinate frame $\left(\mathrm{O}^{\prime}, \mathrm{X}^{\prime}, \mathrm{Y}^{\prime}, \mathrm{Z}^{\prime}\right)$. The translation along the endoscope path $\mathrm{ABC}$ is of the order of $20 \mathrm{~cm}$.

To quantify the forces and torques during operations, except participation in actual operations, a sensitive force scale apparatus, was built and used by surgeons, see Fig 3 . The measured maximum values of the endoscopeimposed forces and torques are very small as shown in Table 2. To be able to reproduce such small forces faithfully, the haptic mechanism must satisfy strict requirements such as low inertia, low friction, low backlash and back driveability. For accurate emulation of the haptic feeling, the device must refresh forces and torques with a frequency close to $1 \mathrm{kHz}$. In addition, the mechanism must not load the endoscope with its own weight. To achieve this, either the mechanism actuators would have to provide compensating torques, or the mechanism would have to be statically balanced. The latter was chosen as is discussed in more detail next.

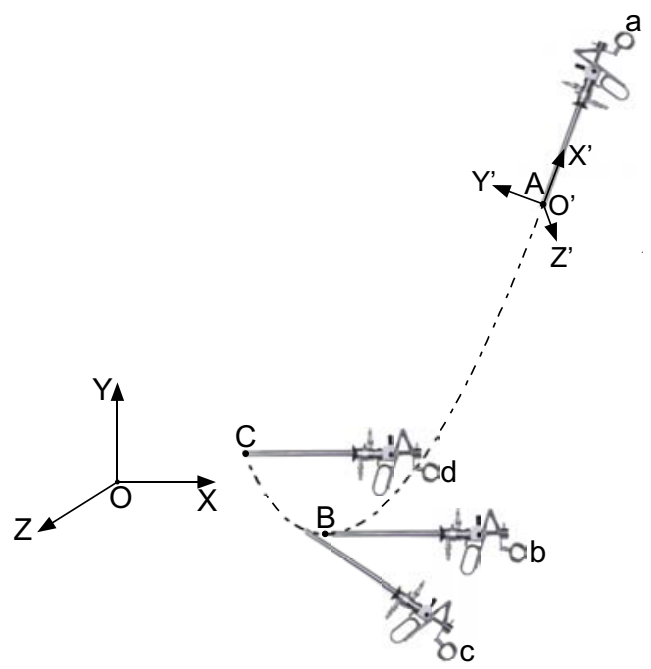

Fig. 2. Endoscope path during an operation.

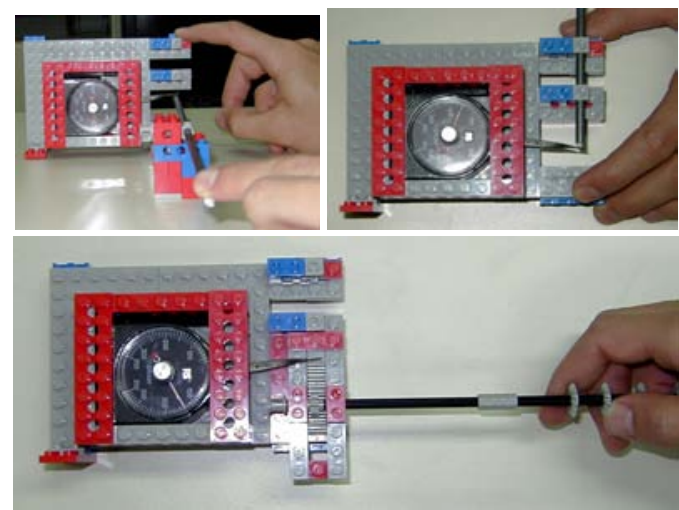

Fig. 3. Experimental force and torque estimation.

Table 1. Haptic device workspace requirements.

\begin{tabular}{lc}
\hline Translation along the $X$ axis & $10 \mathrm{~cm}$ \\
\hline Translation along the $Y$ axis & $10 \mathrm{~cm}$ \\
\hline Translation along the $Z$ axis & $0 \mathrm{~cm}$ \\
\hline Rotation about $X^{\prime}$ axis & $\pm 180^{\circ}$ \\
\hline Rotation about $Y^{\prime}$ axis & $\pm 30^{\circ}$ \\
\hline Rotation about $Z^{\prime}$ axis & $\pm 30^{\circ}$ \\
\hline
\end{tabular}

Table 2. Maximum values of imposed forces and torques during a urological operation.

\begin{tabular}{ll}
\hline Force along the $\mathrm{X}$ axis & $1.5 \mathrm{~N}$ \\
\hline Force along the $\mathrm{Y}$ axis & $1.5 \mathrm{~N}$ \\
\hline Torque about the $\mathrm{X}^{\prime}$ axis & $2.0 \mathrm{mNm}$ \\
\hline Torque about the $\mathrm{Y}^{\prime}$ axis & $70.0 \mathrm{mNm}$ \\
\hline Torque about the $\mathrm{Z}^{\prime}$ axis & $70.0 \mathrm{mNm}$ \\
\hline
\end{tabular}




\section{KINEMATICS \& DYNAMICS}

Kinematics. A hybrid design was developed that satisfies in principle the interface requirements. It consists of a 5-bar parallel linkage with two translational dofs in series to a spherical joint with three rotational dofs, as depicted schematically in Fig. 4. The endoscope is attached at point $\mathrm{E}$ in Fig. 4, i.e. the point at which the three axes of the last three joints intersect. For design reasons, both the differential kinematics and the dynamics of the mechanism are important and developed next.

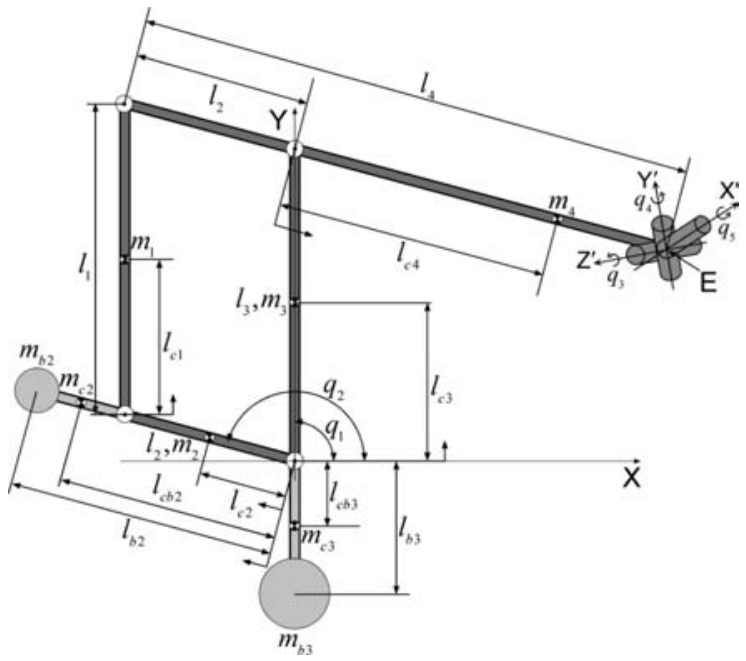

Fig. 4. Schematic view of a 5 dof haptic mechanism. Light gray parts correspond to balancing.

The differential kinematics for this mechanism relates the endpoint Cartesian velocities and angular velocities $\mathbf{v}$ to joint rates $\dot{\mathbf{q}}$

$$
\begin{gathered}
{\left[\begin{array}{c}
\dot{x} \\
\dot{y} \\
\omega_{x} \\
\omega_{y} \\
\omega_{z}
\end{array}\right]=\left[\begin{array}{ccccc}
-l_{3} s_{1} & l_{4} s_{2} & 0 & 0 & 0 \\
l_{3} c_{1} & -l_{4} c_{2} & 0 & 0 & 0 \\
0 & 0 & 0 & s_{2+3} & -c_{2+3} c_{4} \\
0 & 0 & 0 & -c_{2+3} & -s_{2+3} c_{4} \\
0 & 1 & 1 & 0 & -s_{4}
\end{array}\right]\left[\begin{array}{l}
\dot{q}_{1} \\
\dot{q}_{2} \\
\dot{q}_{3} \\
\dot{q}_{4} \\
\dot{q}_{5}
\end{array}\right]} \\
\mathbf{v}=\mathbf{J}_{1}(\mathbf{q}) \dot{\mathbf{q}}
\end{gathered}
$$

where $s_{i}$ is the $\sin ($.$) of angle q_{i}, c_{i}$ is the $\cos ($.$) of angle$ $q_{i}, s_{i+j}$ is the $\sin ($.$) of angle q_{i}+q_{j}$, etc.

The translational five bar mechanism becomes singular when $q_{1}=q_{2}$ or $q_{1}=q_{2} \pm \pi$. The spherical joint, which is of the $\mathrm{z}-\mathrm{y}-\mathrm{x}$ type, is singular when $q_{4}= \pm \pi / 2$. However, all these configurations will not be possible to the actual mechanism due to its design.

The joint rates are related to actuator rates $\dot{\boldsymbol{\theta}}$ via Jacobian $\mathbf{J}_{2}$,

$$
\dot{\mathbf{q}}=\mathbf{J}_{2}\left[\dot{\theta}_{1}, \dot{\theta}_{2}, \dot{\theta}_{3}, \dot{\theta}_{4}, \dot{\theta}_{5}\right]^{T}=\mathbf{J}_{2} \dot{\boldsymbol{\theta}}
$$

which depends on the transmission technique used. Then, the endpoint velocities are written as

$$
\mathbf{v}=\mathbf{J}_{1}(\mathbf{q}) \mathbf{J}_{2} \dot{\boldsymbol{\theta}}=\mathbf{J}(\mathbf{q}) \dot{\boldsymbol{\theta}}
$$

Dynamics. Employing a Lagrangian formulation yields the following mechanism equations of motion

$$
\mathbf{M}(\mathbf{q}) \ddot{\mathbf{q}}+\mathbf{V}(\mathbf{q}, \dot{\mathbf{q}})+\mathbf{G}(\mathbf{q})=\boldsymbol{\tau}^{\prime}+\mathbf{J}_{1}{ }^{T} \mathbf{F}_{T}
$$

In Eq. (4), $\mathbf{M}(\mathbf{q})$ is the mechanism $5 \times 5$ mass matrix, $\ddot{\mathbf{q}}$ is the joint acceleration vector, $\mathbf{V}(\mathbf{q}, \dot{\mathbf{q}})$ is a vector, which contains the nonlinear velocity terms, and $\mathbf{G}(\mathbf{q})$ is the gravity torques vector. The vector $\tau^{\prime}$ contains joint input torques, while the vector $\mathbf{J}_{1}^{T} \mathbf{F}_{T}$ resolves the forces and torques applied by the endoscope at the mechanism endpoint, to the five joints.

For control reasons, it is useful to eliminate the gravitational terms and choose the mass distribution such that the mass matrix is a constant matrix.

The mass matrix diagonal terms are independent of the mechanism configuration $\mathbf{q}$ assuming that the spherical joint center of mass coincides with the axes intersection

$$
\mathbf{M}(\mathbf{q})=\left[m_{i j}\right], m_{i i}=\text { const }
$$

The upper two non-diagonal terms can be eliminated by setting

$$
m_{1} l_{c 1} l_{2}=l_{3}\left(m_{4} l_{c 4}+l_{4-2}\left(m_{5}+m_{6}+m_{7}\right)\right)
$$

where $l_{4-2}=l_{4}-l_{2}$.Under the same assumption, the nonzero gravitational static terms for the unbalanced mechanism are

$$
\begin{gathered}
g_{1}(\mathbf{q})=g \mathrm{c}_{1}\left(m_{1} l_{c 1}+m_{3} l_{c 3}+\left(m_{4}+m_{5}+m_{6}+m_{7}\right) l_{3}\right) \\
g_{2}(\mathbf{q})=g \mathrm{c}_{2}\left(m_{1} l_{2}+m_{2} l_{c 2}-m_{4} l_{c 4}-\left(m_{5}+m_{6}+m_{7}\right) l_{4-2}\right)
\end{gathered}
$$

These terms can be eliminated if two balancing weights are used with masses $m_{b 2}$ and $m_{b 3}$, given by

$$
\begin{aligned}
& m_{b 3}=\frac{1}{l_{b 3}}\left(m_{3} l_{c 3}+\left(m_{4}+m_{5}+m_{6}+m_{7}\right) l_{3}-m_{c 3} l_{c b 3}+m_{1} l_{c 1}\right) \\
& m_{b 2}=\frac{1}{l_{b 2}}\left(m_{4} l_{c 4}+\left(m_{5}+m_{6}+m_{7}\right) l_{4-2}-m_{1} l_{2}-m_{c 2} l_{c b 2}-m_{2} l_{c 2}\right)
\end{aligned}
$$

and located as shown in the light gray shaded part of Fig. 4. Although other locations for these do exist, the chosen ones minimize the effective mechanism inertia and are easier to implement.

Using Eq. (1b) and its derivative,

$$
\ddot{\mathbf{q}}=\mathbf{J}_{1}^{-1}\left(\dot{\mathbf{v}}-\dot{\mathbf{J}}_{1} \mathbf{J}_{1}^{-1} \mathbf{v}\right)
$$

and assuming a balanced mechanism, the equations of motion as seen from the endoscope side and written for its tip motion reduce to

$$
\tilde{\mathbf{M}} \dot{\mathbf{v}}+\tilde{\mathbf{V}}=\mathbf{J}^{-T} \boldsymbol{\tau}+\mathbf{F}_{T}
$$

with

$$
\tilde{\mathbf{M}}=\mathbf{J}_{1}^{-T} \mathbf{M} \mathbf{J}_{1}^{-1}, \quad \tilde{\mathbf{V}}=\mathbf{J}_{1}^{-T} \mathbf{V}-\mathbf{J}_{1}^{-T} \mathbf{M} \mathbf{J}_{1}^{-1} \dot{J}_{1} \mathbf{J}_{1}^{-1} \mathbf{v}
$$

where $\tau$ is the motor torque vector. Notice that the effective mass matrix $\tilde{\mathbf{M}}$ is not constant due to the multiplication with the Jacobian. Although it is not possible to make $\tilde{\mathbf{M}}$ constant, its structure is simpler than if $\mathbf{M}$ were not a constant.

\section{MECHANISM IMPLEMENTATION}

The major challenges in implementing the mechanism that was chosen include inertia and friction minimization, 
balancing and most importantly, transmission of forces and torques. Although some of these issues are important in other haptic devices, here they are of paramount importance given the small forces that must be reproduced faithfully by the mechanism. Next, some of the design criteria used are discussed.

Translational Mechanism. The dimensions of the five bar mechanism depend on the typical path and its location. Additional constraints exist which limit the mechanism dimensions. (a) To satisfy the criteria of minimum mass and inertia, the mechanism must not be larger that needed. (b) To avoid singularities and closeness to them, the mechanism condition number that is computed using its Jacobian must be minimum. It is found condition number is minimum when $l_{4}-l_{2}=l_{3}$ and $q_{2}-q_{1}=90^{\circ}$. Additional mechanical constrains exist which depend on mechanism geometry. For example, to avoid misplacement of the ropes, the relative angle between the tool and link 4 should be less than $\pm 100^{\circ}$, or the angle between links 2 and 3 cannot be less than $30^{\circ}$. These constraints are conflicting to some extent. For example use of length ratios that yield an optimal condition number results in a larger than needed mechanism violating the minimum mass and inertia criterion.

For this mechanism, the typical average path to be followed by the endoscope tip is shown in Fig. 2. The actual path differs slightly due to tissue deformation and to small parametric changes that account for different anatomies. Therefore, it is important to find the best location of the path with respect to the mechanism base, or equivalently, given the path $\mathrm{ABC}$ in Cartesian space, to find where the mechanism base should be. To answer this question, the path $\mathrm{ABC}$ was drawn in the mechanism's workspace with a varying start point so that the entire path could be followed by the mechanism given the above described constraints. For example, since the angle between links 2 and 3 cannot be less than $30^{\circ}$, this excludes all starting points $\mathrm{A}$ for which this angle falls below $30^{\circ}$ at some point during the execution of the path. Of the feasible paths, the best was chosen by taking the condition number at the end of the path to be minimum. The location of the chosen path yielded the link lengths tabulated in Table 3 .

Table 3. Links lengths

\begin{tabular}{lc}
\hline Link $1, l_{1}$ & $13.50 \mathrm{~cm}$ \\
\hline Link $2, l_{2}$ & $7.50 \mathrm{~cm}$ \\
\hline Link $3, l_{3}$ & $13.50 \mathrm{~cm}$ \\
\hline Link $4, l_{2}+l_{4}$ & $23.00 \mathrm{~cm}$ \\
\hline
\end{tabular}

Figure 5 shows some locations for the typical path in the mechanism workspace, the condition number diagram along the paths shown and the condition number diagram for the optimally located path. As pointed out above, fixing the path in space yields the location of the base of the mechanism, which is very useful in completing its design.
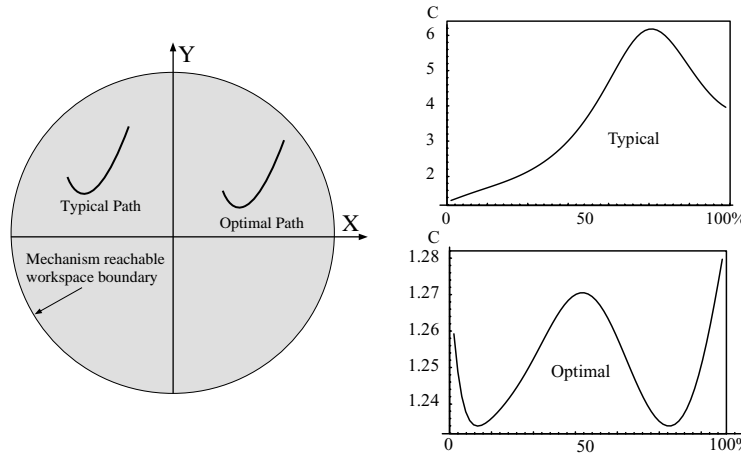

Fig. 5. Optimal location of the required path.

Spherical joint description. Designing a spherical joint is a challenge, especially if the joint must be actuated. The actuators can be either on the mechanism, or at the non-moving base. The first option increases the mass and inertia of the mechanism and therefore the second option is preferable, provided that the transmission mechanism is properly designed and does not introduce high friction, backlash or other nonlinearities. In addition, the orientational dofs should be designed such that their motions were decoupled, i.e., a single roll, pitch or yaw rotation should affect one motor rotor.

The decoupling of the spherical joint is necessary for two reasons. The first is that the Jacobian matrix should be relative simple. This is of great importance because it allows to keep the control loop as fast as possible. The second reason has to do with the fact that during the operation, the surgeon tends to operate in decoupled rollpitch-yaw motions. This is especially true for roll, i.e. rotation around the endoscope axis. In a decoupled rollpitch-yaw design, these motions correspond to rotations of one drive system at a time, and therefore the felt inertia is also minimized.

Due to the relatively limited range of joint motions and to transmission design reasons, wire capstan drives were chosen. Their advantages include the absence of backlash and of radial bearing loads. Low stretch Dacron rope was used and was guided via miniature ball-bearing idler pulleys as described below. To design an effective spherical joint based on such transmissions, the lengths of the ropes must not change during any rotation or translation. In addition, when one of the roll-pitch-yaw motions is in progress, only the ropes of the relevant dof must move.

To keep the lengths of the rotational dof ropes constant during translational movements of the mechanism, the ropes are wrapped and unwrapped around different pulleys by the same angle.

To transmit the motion to the rotational dof and keep the total rope length constant during rotational motions of the previous rotational dof, the ropes must pass from points that do not move during such motions. Such points include intersection of axes, or points located on the axes themselves. Here, the second option was implemented, i.e. the ropes travel along the rotation axis of the previous joint before they leave it.

Figure 6 shows a schematic view of the spherical 
joint design. As shown in the figure, there are three groups of pulleys, the $\mathrm{A}, \mathrm{B}$ and $\mathrm{C}$ groups, which are responsible for rotations $q_{3}, q_{4}$ and $q_{5}$ respectively. Drive pulleys $\mathrm{A}_{1}$ and $\mathrm{A}_{4}$ rotate around the $\mathrm{Z}$ ' axis which is fixed in the five-bar mechanism and therefore the length of the wires for angle $q_{3}$ is always constant.

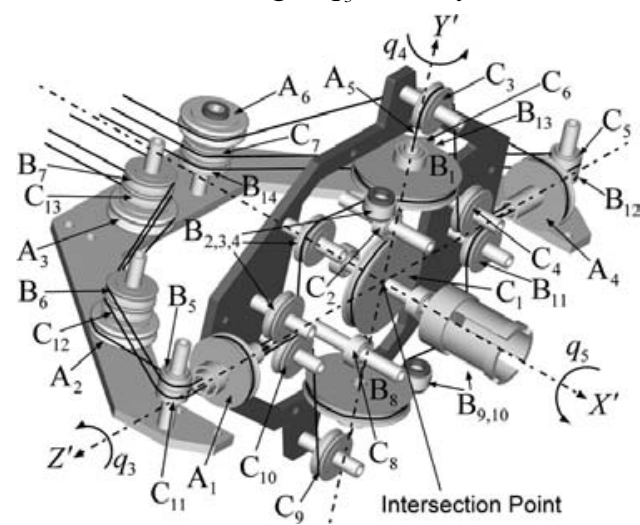

Fig. 6. Schematic view of the 3-dof spherical joint and idler pulleys.

Idler pulleys sets $\mathrm{B}_{2}, \mathrm{~B}_{3}, \mathrm{~B}_{4}$ and $\mathrm{B}_{9}, \mathrm{~B}_{10}, \mathrm{~B}_{11}$ receive from drive pulleys $B_{1}$ and $B_{8}$ associated with joint angle $q_{4}$ and guide it on the axis of rotation of the previous joint with angle $q_{3}$ and through the center of drive pulleys $A_{1}$ and $A_{4}$ to idler pulleys $B_{5}$ and $B_{12}$. Similarly, idler pulleys sets $\mathrm{C}_{2}, \mathrm{C}_{3}, \mathrm{C}_{4}$ and $\mathrm{C}_{8}, \mathrm{C}_{9}, \mathrm{C}_{10}$ guide the rope associated with angle $q_{5}$ from drive pulley $\mathrm{C}_{1}$ through the center of drive pulleys $\mathrm{B}_{1}$ and $\mathrm{B}_{8}$ on the axes of the two previous joints. In this way, motions of the two previous joints do not affect the lengths of the ropes for the last joint.

Figure 7 shows the spherical joint assembly. The joint exceeds the design requirements since it permits rotations of $\pm 270^{\circ}$ about the $\mathrm{X}^{\prime}$-axis, $\pm 90^{\circ}$ about the $\mathrm{Y}^{\prime}$ axis, and $\pm 270^{\circ}$ about the $Z^{\prime}$-axis.

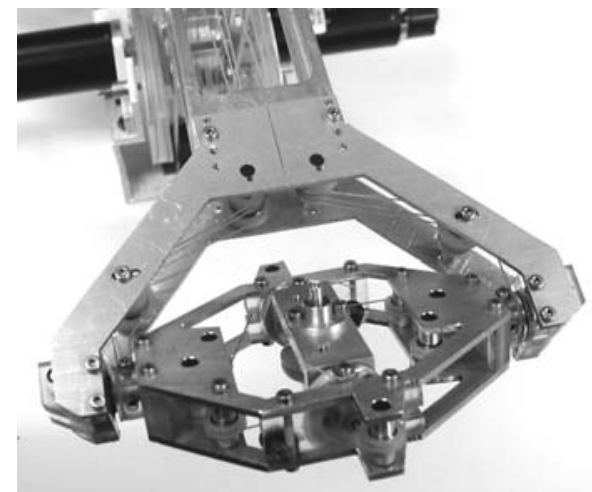

Fig. 7. The assembled 3-dof spherical joint.

Assembled Mechanism. The complete mechanism shown in Fig. 8 was constructed mostly from aluminum. The weight of the moving parts is less than $500 \mathrm{~g}$. The capstan drive transmission rope is under preload for rapid response to input torques. The preload is set equal to the dynamic load, which is the output torque divided by the radius of the output shaft. The $0.5 \mathrm{~mm}$ cable diameter is chosen such that the sum of the preload and dynamic cable loads is less than $10 \%$ of the breaking strength.

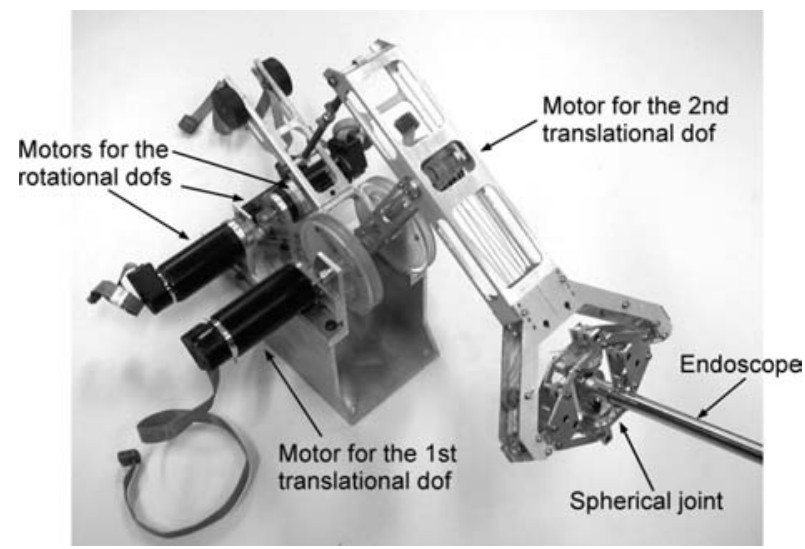

Fig. 8. Assembled haptic device with an endoscope.

For the particular mechanism transmission employed, the transmission Jacobian $\mathbf{J}_{2}$ is given by

$$
\mathbf{J}_{2}=\left[\begin{array}{ccccc}
-r_{1} / R_{1} & 0 & 0 & 0 & 0 \\
0 & -r_{2} / R_{2} & 0 & 0 & 0 \\
0 & r_{2}\left(R_{3,2}-R_{3}\right) / R_{2} R_{3} & r_{3} / R_{3} & 0 & 0 \\
0 & r_{2} R_{4,2} / R_{2} R_{4} & 0 & r_{4} / R_{4} & 0 \\
0 & r_{2} R_{5,2} / R_{2} R_{5} & 0 & 0 & r_{5} / R_{5}
\end{array}\right]
$$

where $R_{i}$ is the radius of the drive pulley of the $i^{t h}$ dof, $r_{i}$ is the radius of the $i^{\text {th }}$ motor capstan, and $R_{i, 2}$ represents the radii of idler pulley for the $i^{\text {th }}$ dof located in the five bar mechanism.

\section{CONTROL}

Coreless DC motors actuate the device and apply torques aiming at giving the feeling that only the endoscope and the tissues are present. To compute the necessary motor currents, the equations of motion of the surgical tool, see Fig. 9, are written as

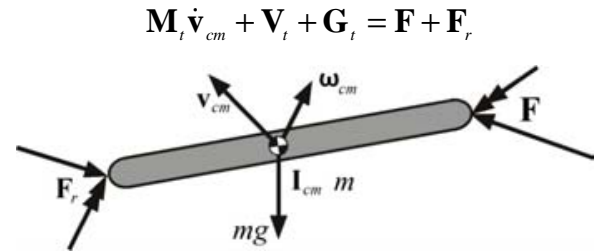

Fig. 9. Forces on the surgical tool.

where $\mathbf{F}$ is the vector of the applied forces and torques by the operator to the tool, $\mathbf{M}_{t}$ is its mass matrix, $\mathbf{V}_{t}$ contains velocity terms, $\mathbf{G}_{t}$ contains gravity terms, and $v_{c m}$ is the velocity of its center of mass. The vector $\mathbf{F}_{r}$ contains forces and torques which are due to tissue deformation. Since no motion is allowed in the $\mathrm{Z}$ direction, in the virtual environment, $\mathbf{M}_{t}$ is a $5 \times 5$ matrix and the rest of the vectors have appropriate dimensions.

The endpoint forces that must be applied by the tool to the mechanism are $\mathbf{F}_{T}=-\mathbf{F}_{r}$ and are given by

$$
\mathbf{F}_{T}=\mathbf{F}-\mathbf{M}_{t} \dot{\mathbf{v}}_{c m}-\mathbf{V}_{t}-\mathbf{G}_{t}
$$

Replacing $\mathbf{F}_{T}$ given by Eq. (13) to Eq. (10a) yields 


$$
\mathbf{M}_{t} \dot{\mathbf{v}}_{c m}+\mathbf{V}_{t}+\mathbf{G}_{t}=\mathbf{F}+\mathbf{J}^{-T} \boldsymbol{\tau}-\tilde{\mathbf{M}} \dot{\mathbf{v}}-\tilde{\mathbf{V}}
$$

In order that the presence of the haptic device is not felt, the right sides of Eqs. (12) and (14) must be identical. Then, the actuator torques must be equal to

$$
\boldsymbol{\tau}=-\mathbf{J}^{T} \mathbf{F}_{T}+\tilde{\mathbf{M}} \dot{\mathbf{v}}+\tilde{\mathbf{V}}
$$

To a first approximation, it is assumed that the accelerations and velocities are very small, and hence, the last two terms in Eq. (15) can be neglected. At a later stage, the most prominent mechanism inertial force terms, as well as neglected friction terms, will be taken into account. With these assumptions, the required torques are

$$
\boldsymbol{\tau}=-\mathbf{J}^{T} \mathbf{F}_{T}
$$

In general, the forces and torques $\mathbf{F}_{T}$ are functions of the position and velocity of the tool and are computed based on a simplified model of tissue deformation as

$$
\mathbf{F}_{T}=\mathbf{F}_{T}(\dot{\mathbf{q}}, \mathbf{q})
$$

Finally, the currents that are sent to the motors by the power amplifiers are given by

$$
\mathbf{i}=-\left(\mathbf{K}_{\mathbf{T}} \mathbf{K}_{\text {amp }}\right)^{-1} \mathbf{J}^{T} \mathbf{F}_{T}(\dot{\mathbf{q}}, \mathbf{q})
$$

where $\mathbf{K}_{\mathrm{T}}$, and $\mathbf{K}_{a m p}$ are diagonal matrices that contain motor torque constants and amplifier gains, respectively.

The haptic device is part of a training simulator that includes a visual display displaying a synthetic endoscope camera view. To increase the speed of the force loop, two separate processes run on different platforms. The control loop and the calculation of the forces and torques that the user should feel are implemented in a stack of $\mathrm{PC} / 104$ cards which consist of a $233 \mathrm{MHz}$ CPU module, a $24 \mathrm{MB}$ solid state flash disk, an Ethernet communications module, two encoder polling input modules, and an analog output module, which drives the motor amplifiers, see Fig. 10a. In order to have a constant refresh rate, $\mathrm{QNX}^{\mathrm{Q}}$, a real time operating system from QNX Software Systems Ltd., is employed.

The visual display of the endoscope view of the tissues and their deformations is produced by a separate process running on a Pentium III computer equipped with the OpenGL graphic library. This process reads from the $\mathrm{PC} / 104$ stack the position, orientation and velocity of the surgical tool, see Fig. 10b. The processes communicate with each other via Ethernet cards and the TCP/IP protocol.

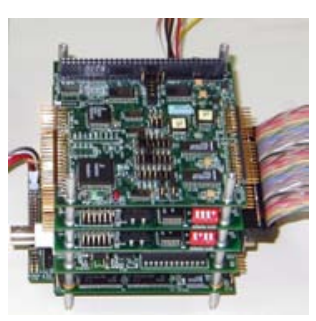

(a)

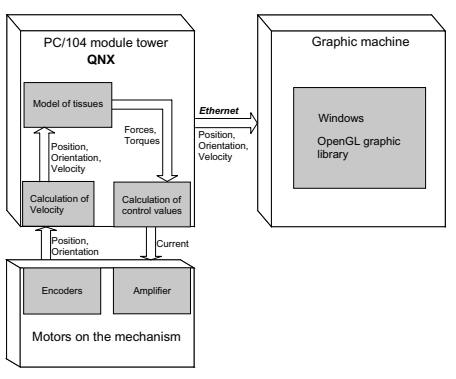

(b)
Fig. 10. (a) PC/104 stack. (b) Software / Hardware configuration.

\section{CONCLUSIONS}

A new force feedback mechanism is presented that is used in a training simulator for urological operations. The mechanism consists of a two dof, 5-bar linkage for translations and a 3-dof spherical joint for rotations. To be able to reproduce very small forces and moments, the mechanism has low friction, inertia and mass, is statically balanced, and has a simple mass matrix by design. To minimize the mass of its moving parts, the actuators are located at its base and forces and moments are transmitted through the use of capstan drives and idler pulleys. Roll-pitch-yaw motions of the tool result in motions of the corresponding actuator. The currents needed to simulate tissue deformation forces and moments were computed and the implementation of the control loop running at $1 \mathrm{kHz}$ was described.

\section{ACKNOWLEDGMENTS}

Support of this work by the PENED programs of the Hellenic General Secretariat for Research and Technology is acknowledged. The authors also wish to thank P. Hatzakos and L. Bahas for their assistance in the CAD drawings and mechanism realization.

\section{REFERENCES}

[1] Sorid D. and Moore K. S., The Virtual Surgeon, in IEEE Spectrum, Jul. 2000, vol. 37, no. 7, pp. $26-31$.

[2] Chen E., Marcus B., Force Feedback for Surgical Simulation, Proc. of the IEEE, Mar. 1998, vol. 86, no. 3, pp. 524-530.

[3] Goertz R. C., Fundamentals of General - Purpose Remote Manipulators, Nucleonics, 1952, Vol. 10, No. 11, pp. 36 - 42.

[4] Salisbury J. K. and Srinivasan A. M., Projects in VR. Phantom Based Haptic Interaction with Virtual Objects, IEEE Computer Graphics and Applications, Sep/Oct 1997, pp. 6- 10

[5] D Aulignac D., Balaniuk R., and Laugier C., A Haptic Interface for a Virtual Exam of the Human Thigh, Proc. IEEE Int. Conference on Robotics and Automation, April 2000, pp. 2452 2457.

[6] Atsuko T., Koichi H., and Toyohisa K., Virtual Cutting with Force Feedback, Proc. of the Virtual Reality Annual International Symposium, 1998, pp. $71-75$.

[7] Massie T. H. and Salisbury J. K., The PHANToM Haptic Interface: A Device for Probing Virtual Objects, Proc. of the 1994 ASME Int. Mechanical Engineering Exposition and Congress, Chicago, Illinois, 1994, pp. 295 - 302.

[8] Chen E., Six Degree-of-Freedom Haptic System for Desktop Virtual Prototyping Applications, Proc. First International Workshop on Virtual Reality and Prototyping, June 1999, pp. 97106, Laval France.

[9] Burdea G., et al., Virtual Reality — based Training for the Diagnosis of Prostate Cancer, IEEE Transactions on Biomedical Engineering, Vol. 46, No 10, October 1999, pp. 1253 - 1260.

[10] Hayward V., et al., Freedom — 7: A High Fidelity Seven Axis Haptic Device With Application To Surgical Training, Experimental Robotics V, Casals, A., de Almeida, A. T. (eds.), Lecture Notes in Control and Information Science 232, 1998, pp. $445-456$.

[11] Baumann R., et al., The PantoScope: A Spherical Remote Center - of - Motion Parallel Manipulator for Force Reflection, in Proc. IEEE Int. Conference on Robotics and Automation, April 1997, pp. $718-723$.

[12] Baur C., et al., A Virtual Reality and Force Feedback Based Endoscopy Surgery Simulator, in Proceedings - Medicine Meets Virtual Reality 98, (MMVR 98), 1998, pp. 110-116.

[13] K hnapfel U., et al., The Karlsruhe Endoscopic Surgery Trainer as an example for Virtual Reality in Medical Education, in Minimally Invasive Therapy and Allied Technologies (MITAT), Blackwell Science Ltd,, 1997, pp. 122-125. 